\title{
New method for preserving cytology specimens
}

\author{
J McCOR RISTON Cytology Department, Royal Infirmary, Glasgow, Scotland
}

SUMMARY A method of freezing cytological material for long term storage, based on a modification of that used in tissue typing for the storage of lymphocytes, was developed. The method entails the centrifugation of the specimen, adding $1 \mathrm{ml} \mathrm{AB}$ or fetal calf serum and $1 \mathrm{ml} 20 \%$ dimethyl sulphoxide aliquoted into $2 \mathrm{ml}$ tubes, and storage at $-70^{\circ} \mathrm{C}$. Cytological detail was well preserved in a variety of samples including fine needle aspirates, urine, and bronchial brushings. Three dimensional architecture was also preserved.

The method is practical, easy to perform, and allows retrospective studies to be undertaken with the subsequent use of a variety of special staining techniques, including immunocytochemistry.

Cytology has the principal disadvantage that, unlike histology, additional preparations cannot be made other than at the time of initial preparation. While samples can be preserved by processing to paraffin wax, the cytological nature of the specimen is lost. Alternatively, slide preparations can be stored in a refrigerator for subsequent analysis, but this can be time consuming and difficulties may arise when insufficient slides have been made. In an attempt to overcome these problems a method of freezing cytological material for long term storage was developed. It was considered essential that both cellular architecture and nuclear morphology were preserved. The ability to perform special stains, including immunocytochemistry was also a prime requirement.

\section{Method}

1 Centrifuge specimen.

2 To the cell button add the following:

AB serum (or fetal calf serum) $1 \mathrm{ml} ; 20 \%$ dimethyl sulphoxide (DMSO) $1 \mathrm{ml}$.

3 Aliquot into $2 \mathrm{ml}$ tubes.

4 Store in $-70^{\circ} \mathrm{C}$ freezer.

Specimens should be frozen slowly as snap freezing can rupture cytoplasmic membranes. Gradual freezing of the specimen is achieved by wrapping the tubes in cotton wool before placing in freezer. As DMSO is cytotoxic at temperatures of greater than $4^{\circ} \mathrm{C}$ it should be stored in a refrigerator before use. Specimens should be reconstituted by rapid thawing and dilution in cold saline as soon as possible. Several saline washes are recommended to remove all traces of DMSO. Specimens must be stored at $-70^{\circ} \mathrm{C}$. Storage at $-4^{\circ} \mathrm{C}$ and $-20^{\circ} \mathrm{C}$ produces inconsistent results. In our laboratory specimens have been stored for up to one year with no detriment.

\section{Discussion}

The method used in this study is a modification of that used in tissue typing where lymphocytes are stored at $-70^{\circ} \mathrm{C}$. ${ }^{1}$ These cells remain viable and are subjected to subsequent histochemical and biochemical analysis.

The method was initially developed to preserve cells from serous effusions (fig 1), but because of the excellent results obtained, it has been applied to a variety of samples including fine needle aspirates, urine, bronchial brushings etc. Cytological detail was well preserved in all cases studied-terminal plates and cilia on bronchial epithelial cells. Three dimensional architecture was also preserved (fig 2).

With this method a wide range of special staining techniques can be used including periodic acid Schiff,

Table Comparison of immunocytochemistry results between fresh and frozen material

\begin{tabular}{lll}
\hline & Reactivity & \\
\cline { 2 - 3 } Antibody used & Fresh & Frozen \\
\hline HMFG 1 & +++ & +++ \\
HMFG 2 & +++ & +++ \\
CEA & +++ & +++ \\
CAM 5.2 & +++ & +++ \\
CLA & +++ & +++ \\
T4 & ++ & ++ \\
T8 & ++ & ++ \\
HLA-DR & ++ & ++ \\
C3b & ++ & + \\
\hline
\end{tabular}




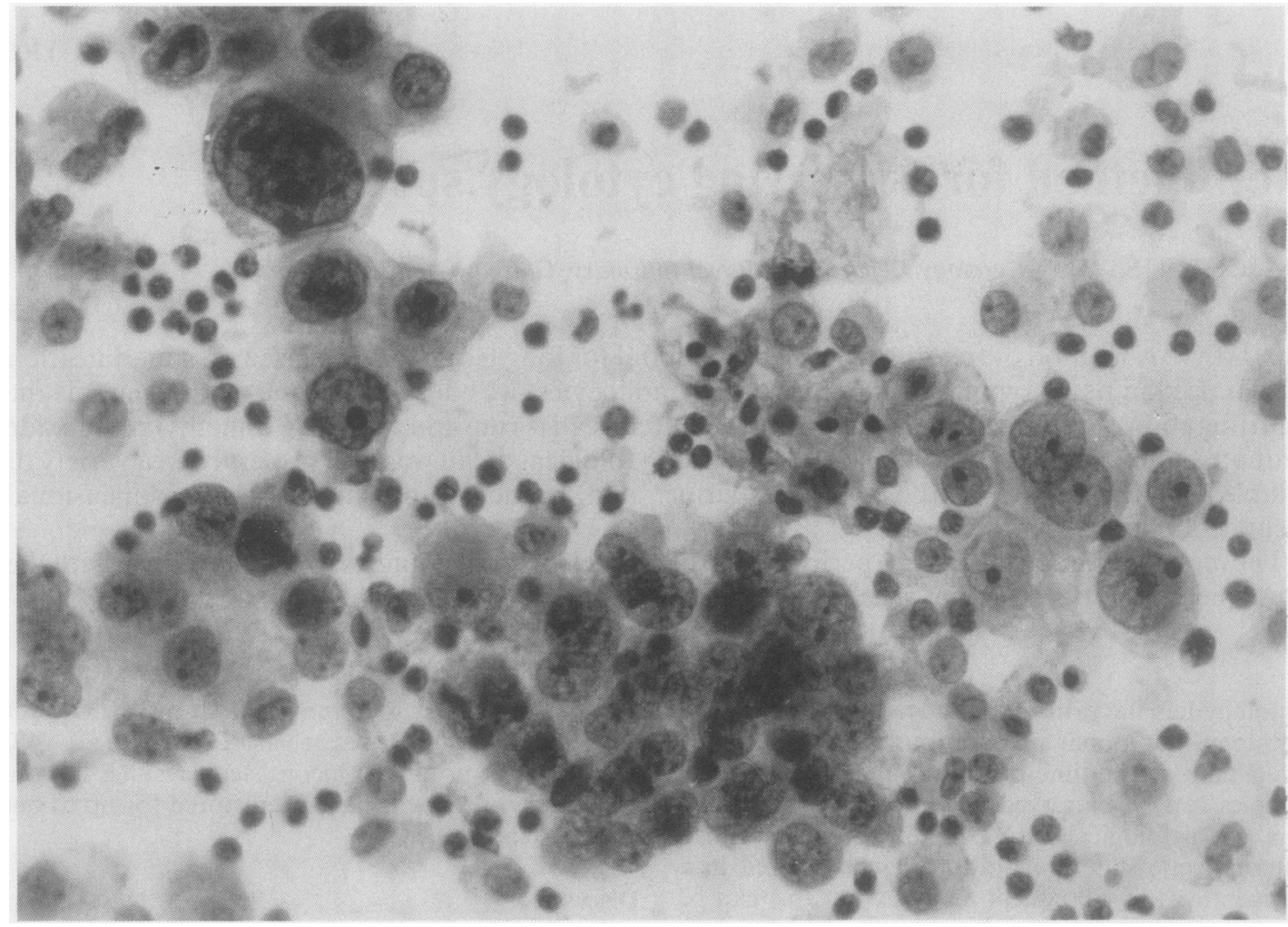

Fig 1 Poorly differentiated malignant cells in a pleural fuid. (Note excellent retention of nuclear and cytoplasmic detail. Storage time-six months. (Papanicolau stain.)

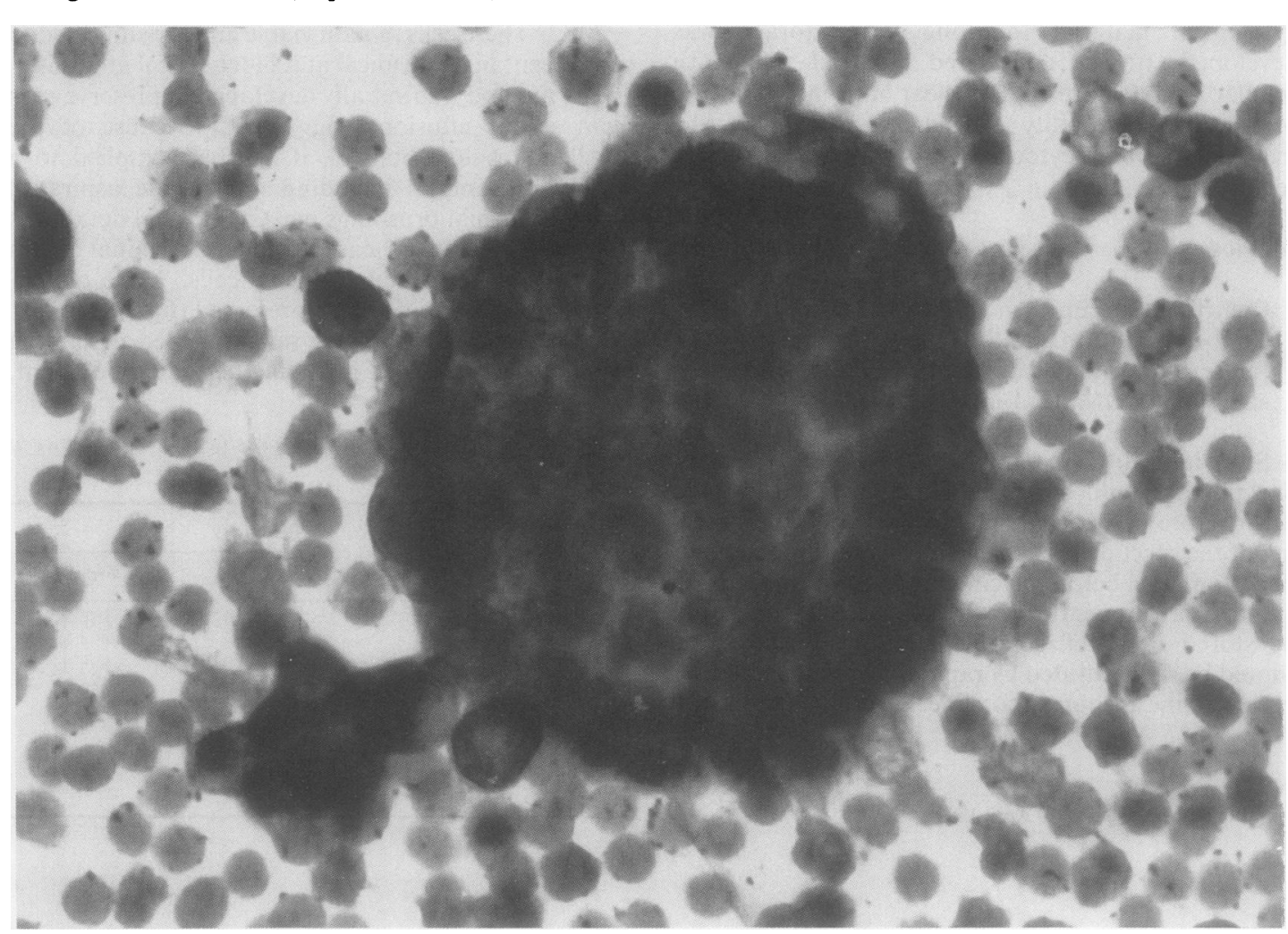

Fig 2 Ball-like cluster of malignant cells in a pleural fluid. Storage time-three months. (Cam 5·2, indirect alkaline phosphatase method). 
Gram Weigert, and silver methods for the demonstration of Pneumocystis carinii. Immunocytochemical techniques can be performed using a variety of antibodies (table).

The results using immunocytochemical techniques were as reliable on frozen as on fresh material. The intensity of staining was not diminished by freezing. There was no change in staining pattern and because of the saline washes done after thawing of the specimen, there was often less background staining. Prolonged storge did not adversely affect the staining reactions.

This study shows that there is an acceptable method for storing specimens which preserves them in a condition suitable for cytological study. It is practical and easy to perform and is applicable to a variety of specimens. It allows retrospective studies to be undertaken and the subsequent use of special staining techniques, including immunocytochemistry. It can be used to preserve interesting cases for teaching material and in the production of cytological control material such as that needed for $P$ carinii.

\section{Reference}

1 Dick HM, Kissmeyer Nielson F. Histocompatibility Techniques. Amsterdam: Elsevier/North Holland; 1979.

Requests for reprints to: Dr J McCorriston, Cytology Department, Royal Infirmary, Castle Street, Glasgow G4, Scotland. 